\title{
Emergency Medicine Physicians' Approaches to Coping with Stress in COVID-19 Pandemic
}

\author{
(D) Mehmet Cihat Demir ${ }^{1}$, (D) Ali Kaan Ataman²
}

1Department of Emergency Medicine, Düzce University, Düzce, Turkey

2Department of Emergency Medicine, Okan University, İstanbul, Turkey

\begin{abstract}
Aim: This study aimed to investigate the stress experienced by emergency medicine physicians working in emergency departments during the coronavirus disease-2019 (COVID-19) pandemic, the factors they stated to be effective against stress, and their coping approaches to stressful situations.

Materials and Methods: The study was designed in a general screening model, and 200 emergency medicine physicians participated via e-mail who work in emergency departments in Turkey. The sources of stress related to the pandemic, the factors that they find effective in combating stress, and their strategies to cope with stress were investigated with relation to their gender, marital status, after-shift accommodation, manner of working in a shift, smoking behavior, having a chronic disease, having children, and spouse's job as a healthcare professional.

Results: While the primary source of stress of emergency medicine physicians during the pandemic was the risk of transmitting the virus to their families, the most influential factor in combating stress was leisure activities. Emergency physicians' approaches to coping with stress were significantly predicted by the variables of using full personal protective equipment while working, having an adequate sleep and resting opportunities, obtaining additional economic income, and not knowing the pandemic's end date.

Conclusion: Emergency medicine physicians used active problem-oriented approaches, and among these, they used the social support seeking approach the most during the pandemic. It is necessary to provide social support, take precautions to care for healthcare workers' families and arrange emergency physicians' shifts to allocate their time to their leisure activities appropriately to reduce stress.
\end{abstract}

Keywords: COVID-19, coping with stress, emergency medicine physician, pandemic, stress

\section{Introduction}

The coronavirus disease 2019 (COVID-19) epidemic, which started in November 2019 in China, caused an increase in the workload, working hours, and healthcare professionals' psychological stress (1). Work-related stress is associated with an overloaded work environment where demand exceeds capacity, and it affects healthcare professionals gravely (2). Emergency medicine physicians (EMPs) on the front line have become very sensitive to physical exhaustion, fear, emotional depression, and sleep problems especially; both due to increased workload and their close contact with infected patients (3). During the COVID-19 pandemic, healthcare professionals work knowing that this is a fatal virus, human-to-human transmission is high, they lack personal protective equipment (PPE), and there is no definitive evidence-based treatment yet (4). Also, physicians' emotional trauma increases even more with the deaths they encounter, including their colleagues (5). In a study by Lai et al. (6); it has been reported that physicians who met the patient first experienced depression, insomnia, and intense anxiety.

Work-related stress has psychological consequences such as mood depression, anxiety, and feelings of helplessness (7). It also has physiological results such as hypertension, cardiovascular disease $(8,9)$. The importance of stress management in the prevention of cardiovascular diseases is emphasized in the guideline (9). It leads 
to undesirable situations such as decreased job satisfaction, decreased productivity and production, and eventually losing experienced personnel (7). When it is evaluated in terms of healthcare professionals, it can be seen that job-related burnout directly affects the quality and safety of the health service provided (10). Determining the sources of stress and the approaches to combating the stress of EMPs, who are working at the forefront during the pandemic, are of great importance.

Although many studies on the stress and anxiety of healthcare professionals before the pandemic, the studies related to the additional load encountered due to the epidemic are limited. It is essential to know the sources of stress and EMPs' strategies to cope with the stress to successfully combat the pandemic that greatly impacted social life and has an unknown end date. Even though the entire community needs to give their best effort, successful public health outcomes are mainly dependent on the effective work of the health workforce (11).

Approaches to coping with stress are addressed in two ways in terms of their functions: the management or alteration of the person-environment relationship that is the source of stress (problem-oriented coping) and the regulation of stressful emotions (emotion-oriented coping) (12). In this study, EMPs' stress management approaches were examined in the context of problem-oriented and emotion-oriented coping. In addition to the individual, institutional and social benefits of the data to be obtained, it will also help define healthcare professionals' stress sources, the factors they stated to effective in combating the stress efficiently, and their approaches to coping with stress. It is even thought that it will help prepare the content and method of preventive and supportive services offered to health professionals.

\section{Materials and Methods}

\section{Study Design and Setting}

This research was planned as a descriptive study in the general screening model to reveal the factors that affect the stress and coping strategies of EMPs working in the emergency departments (EDs) during the COVID-19 pandemic. This study was approved by Düzce University Non-Interventional Clinical Research Ethics Board with the registration number 2020/84.

\section{Selection of Participants}

This research was conducted on EMPs who work in EDs in Turkey during the COVID-19 pandemic. The minimum sample size of the research was calculated as $185(\mathrm{n})$ with the following formula (13): $n=t^{2} p q / d^{2}[t: 1.96 ; p: 0.14, q: 0.86, d: 0.05]$. The data collection tools used in the research were sent to the 276 EMPs' via e-mail. However, 200 EMPs participated in the study (with a response rate of $72.5 \%$ ).

\section{Measurements}

Stress Coping Scale was developed by Folkman and Lazarus, is a 4-point Likert type scale with 66 items, which is frequently used in studies investigating the issue of dealing with stress (12). "Stress Coping Styles Scale", adapted by Sahin and Durak in Turkish, consists of 30 items (14). Sub-dimensions of the scale are self-confident, optimistic, social support seeking, helpless, and submissive approach. The first three of the subscales are called the active problem-oriented. The other two are called passive emotion-oriented. The increase in the scores obtained from the self-confident, optimistic, and social support seeking approach factors of the participants show that they use active styles more in coping with stress. The increase in the scores obtained from the helpless and submissive approach factors indicates that they use passive styles to cope with stress (14). The high scores obtained from the subscales indicate that the sub-scale approach is used more in dealin with stress. However, since the most elevated scores obtained from each subscale differ from each other, corrected scores were calculated in the analysis of the data.

Confirmatory factor analysis was performed to test the construct validity of the scale within the scope of this study and the obtained values were found to be at good and acceptable levels $[2 / \mathrm{SD}=1.523$, RMSEA $=0.051, \mathrm{CFI}=0.903, \mathrm{TLI}=0.882]$ (15). Cronbach's alpha value was 0.84 for problem-oriented, 0.82 for emotion-oriented, 0.79 for self-confident, 0.76 for optimistic, 0.53 for social support seeking, 0.65 for submissive and 0.77 for helpless approach was calculated.

A personal information form was created to determine the participants' demographic information, consisting of questions of age, gender, marital status, having children, the spouse being a healthcare professional, smoking, having a chronic disease, after-shift accommodation, manner of working in a shift.

Pandemic Stress Factors Questionnaire was developed by the researchers to reveal the stress-related factors experienced by the participants during the COVID-19 pandemic. Open-ended questions were included to reveal the stressful situations of EMPs during the pandemic, and which factors they think are useful in coping with stress.

\section{Statistical Analysis}

The data was analyzed using SPSS 17 statistics program, with a 95\% confidence level. Frequency, percentage, mean, median, and standard deviation were used to describe the demographic characteristics of EMPs, the factors that they considered as a source of stress during the COVID-19 pandemic, the factors 
that they stated to be effective in dealing with stress, and their coping approaches. Mann-Whitney $U$, chi-square, and stepwise (forward) multiple regression analyses were conducted for further investigations.

\section{Results}

The average age and shift time of participants was $36.21 \pm 6.16$ years and $18.89 \pm 6.17$ hours, respectively. Detailed demographic characteristics of 200 EMPs who participated in the study were presented in Table 1.

Results towards the approaches of EMPs show that they used the problem-oriented rather than emotion-oriented approach $\left[\bar{X}_{p}=61.8 \pm 14.5 ; \bar{X}_{e}=33.10 \pm 16.15\right]$. Besides, it was found that they resorted to seeking social support mostly $\left[\bar{X}_{p 1}=64.6 \pm 17.5\right]$. Other approaches were self-confident $\left[\bar{X}_{p 2}=62.6 \pm 17.5\right]$, optimistic $\left[\bar{X}_{\mathrm{p} 3}=58.4 \pm 19.5\right]$, helpless $\left[\bar{X}_{\mathrm{e} 1}=33.5 \pm 18.2\right]$ and submissive $\left[\bar{X}_{\mathrm{e} 2}=32.5 \pm 17.9\right]$.

It has been determined that EMPs' problem-oriented $(p=0.020)$ and optimistic $(p=0.015)$ approaches differ significantly in favor of men according to gender (Table 2). Married EMPs had a passive stress approach with a higher average than singles $(p=0.041)$. The submissive stress approach of EMPs remained with their family after their shift was higher than those who remained alone $(p=0.047)$. It was found that the helpless stress approach of the married EMPs is higher than the average of the singles $(p=0.022)$.
Also, the helpless stress approach average of smoker EMPs was higher than that of non-smokers $(p=0.039)$.

The factors that the EMPs stated that they caused stress during the COVID-19 pandemic were transmitting the virus to the family (f:150, 75\%), unknown end date of the pandemic (f:148, 74\%), the risk of self-contamination ( $f: 134,67 \%)$, discomfort from PPE ( $f: 132,66 \%)$, lack of definitive treatment or vaccine ( $f: 119,59.5 \%)$, the necessity of frequent cleaning and equipment change (f:109,54.5\%), lack of full PPE (f:72, 36\%), long shifts (f:60, 30\%), lack of medical equipment ( $f: 58,29 \%)$, having fewer colleagues during shifts (f:55, 27.5\%), and comorbidity (f:22, 11\%).

The research data examining the relationship between stress sources that EMPs experience during the COVID-19 pandemic and demographic variables are presented in Table 3. Accordingly, the risk of transmitting the virus to the family was found to be related to the marital status $(p=0.004)$ and after-shift accommodation $(p=0.009)$. The risk of self-contamination was associated with the manner of working in a shift $(p=0.021)$. It was observed that the comorbidity as a stressor was related to the chronic disease status ( $p=0.000)$, the lack of medical equipment was related to gender $(p=0.019)$, marital status $(p=0.035)$, and chronic disease $(p=0.021)$. The necessity of frequent cleaning and equipment change was associated with gender $(p=0.004)$ and smoking $(p=0.027)$. Besides, the lack of PPE was related to marital status $(p=0.025)$ and the manner of working in a shift $(p=0.034)$.

Table 1. Demographic Characteristics of EMPs Participated to the Study

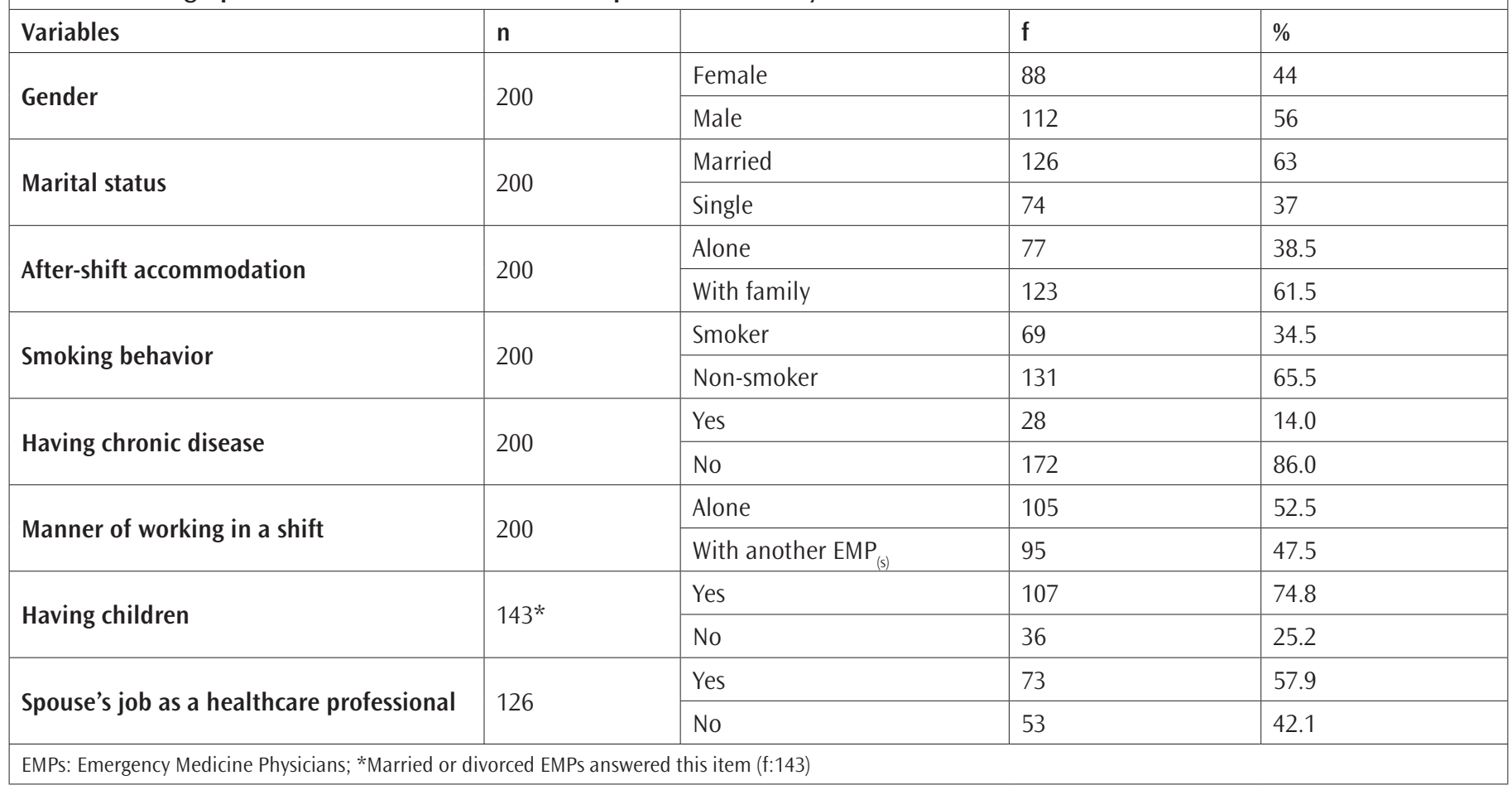




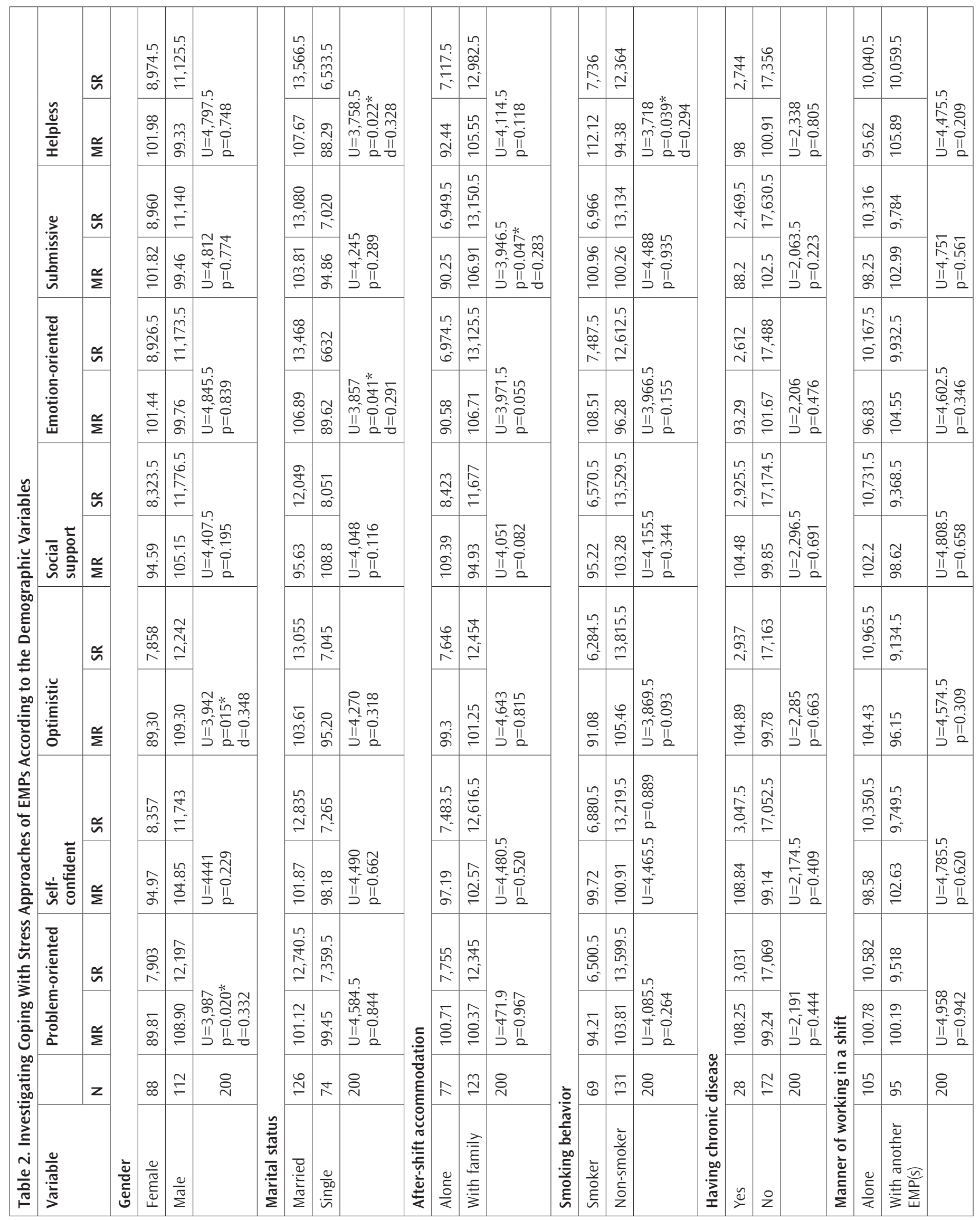




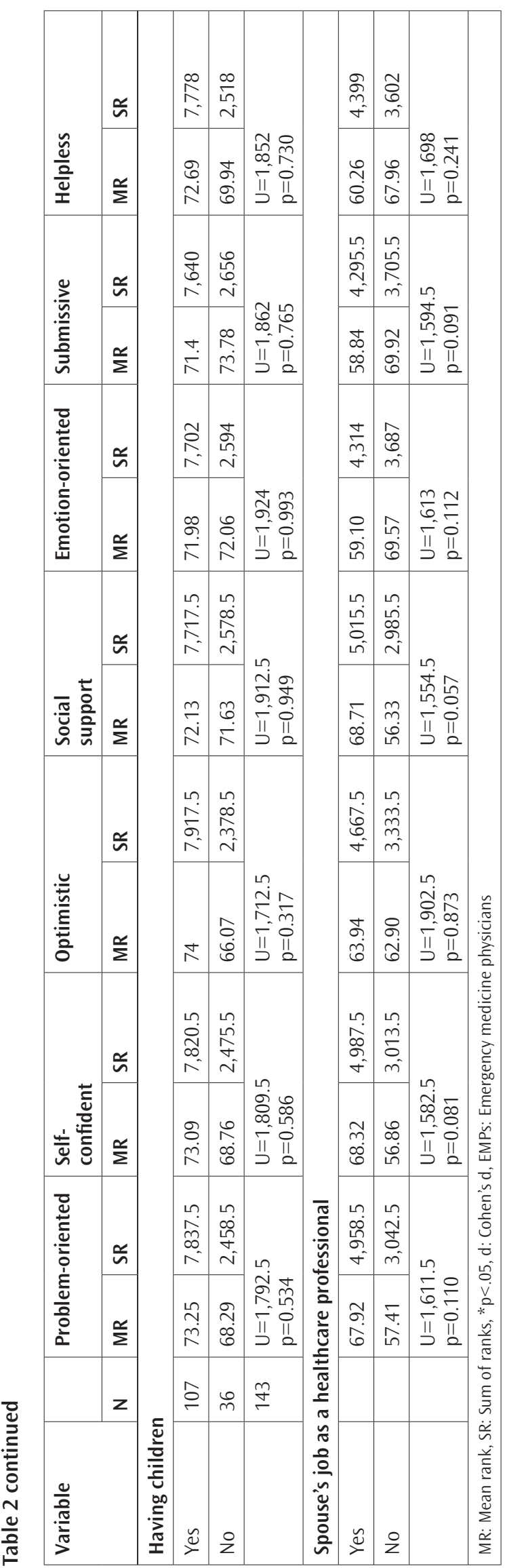

There was a significant relation between long shifts and marital status ( $p=0.030$ ), after-shift accommodation ( $p=0.029)$, manner of working in a shift $(p=0.009)$, having children $(p=0.004)$, and spouse's health professional status ( $p=0.038$ ). Lack of co-worker was found related to after-shift accommodation $(p=0.026)$, manner of working in a shift $(p=0.010)$ and having children $(p=0.007)$. Besides, the unknown end date of the pandemic was found related to gender $(p=0.010)$. A significant correlation was found between the lack of definitive treatment or vaccine and gender $(p=0.002)$.

The factors that EMPs stated that they were effective in dealing with the stress they experienced during the COVID-19 pandemic were mostly leisure activities (f:124, 62\%), having full PPE while working (f:106, 53\%), additional income (f:96, 48\%), public appreciation (f:83, 41.5\%), having adequate sleep and rest (f:74, 37\%), religion (f:22, 11\%) and psychological support (f:18, 9\%). Also, as seen in Table 4, the appreciation was found to be associated with having children $(p=0.012)$ and spouse's being a healthcare professional $(p=.009)$. Getting psychological support was linked to having a chronic disease $(p=0.025)$.

The regression analysis result showed that having full PPE while working and having adequate sleep/rest were significant predictors and explained $5.6 \%$ of the total variance in the active coping approaches of EMPs for stress $(p=0.003)$ (Table 5). It was concluded that having full PPE while working was a significant predictor and explained $6 \%$ of the total variance in EMPs' selfconfidant coping approach to stress $(p=0.000)$. It was determined that having adequate sleep/rest was a significant predictor and explained 2.6\% of the total variance in EMPs' optimistic approach to coping with stress $(p=0.022)$. Besides, the additional income was a significant predictor and explained $2.2 \%$ of the level of EMPs resorting to social support seeking approach to deal with stress $(p=0.037)$.

According to the multiple regression analysis results, it was concluded that the unknown end date of the pandemic, additional income, and having full PPE variables were significant predictors and explained $7.5 \%$ of the total variance in the EMPs' passive coping approach to stress $(p=0.002)$. Additionally, it was decided that the pandemic's unknown end date, additional income, and having full PPE while working were significant predictors and explained $8.7 \%$ of the total variance in EMPs' helpless coping approach to stress $(p=0.000)$. However, as a result of forward stepwise regression analysis that revealed the variables predicting EMPs' submissive coping approach to stress, a significant model and independent variable could not be calculated. 


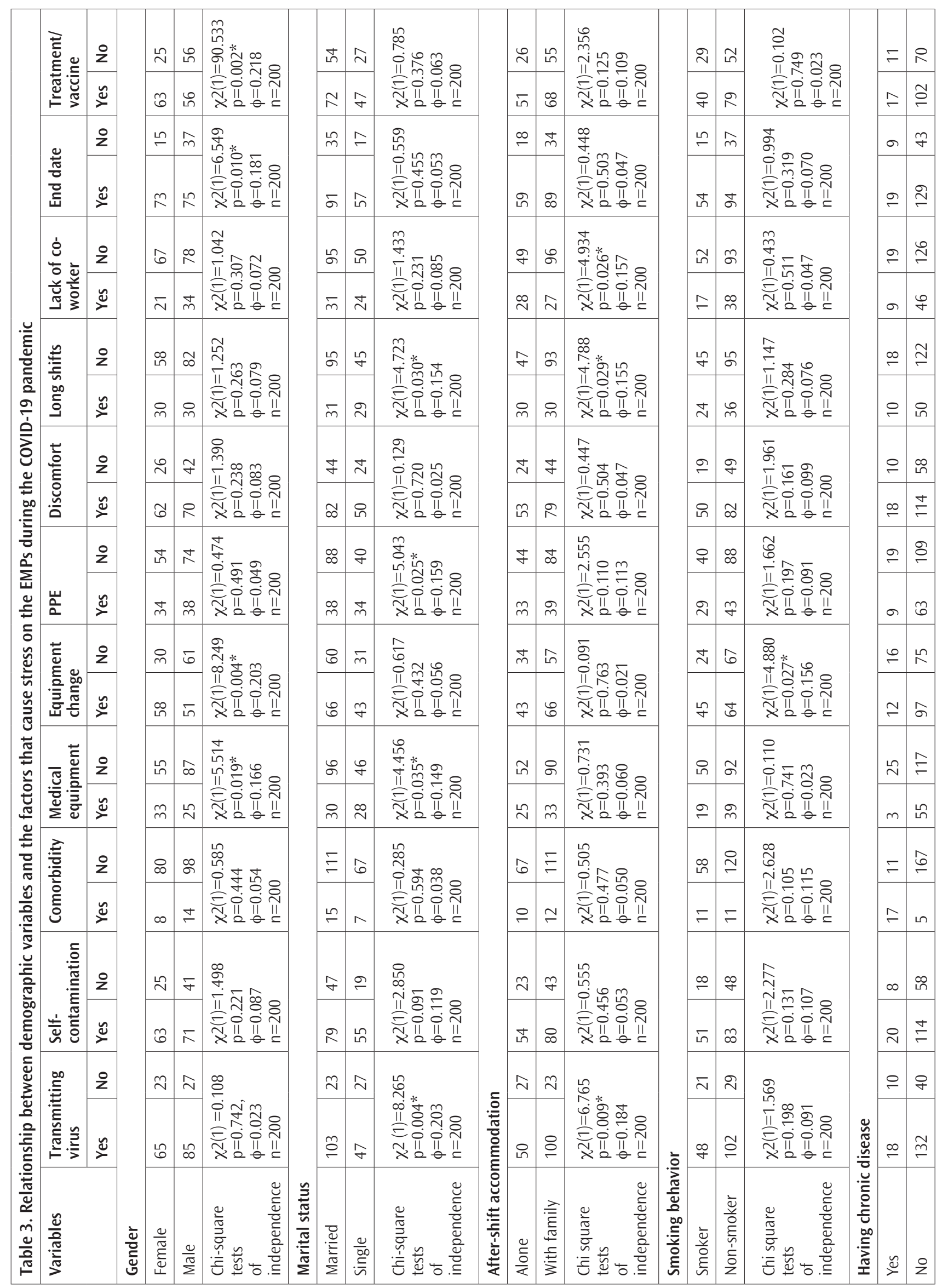




\begin{tabular}{|c|c|c|c|c|c|c|c|c|c|c|c|c|c|c|c|}
\hline 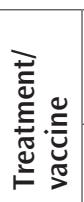 & $\stackrel{\check{\nu}}{\check{\nu}}$ & 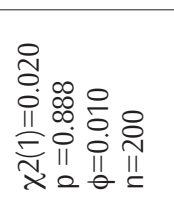 & & $\hat{\sigma}$ & กิ & 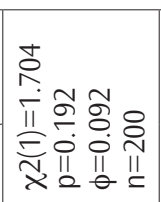 & & 6 & $\bar{v}$ & 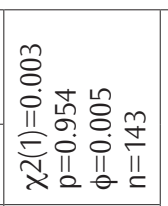 & & J & $\stackrel{\infty}{\sim}$ & 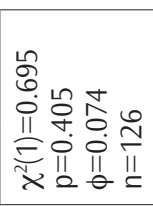 & 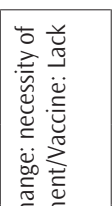 \\
\hline $\begin{array}{l}\frac{\pi}{\frac{\pi}{0}} \\
\frac{0}{5}\end{array}$ & $\stackrel{\widetilde{\nu}}{\nu}$ & 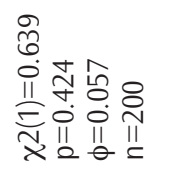 & & $\stackrel{\infty}{\Gamma}$ & $R$ & 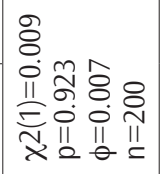 & & $\stackrel{0}{R}$ & $\stackrel{\infty}{\sim}$ & 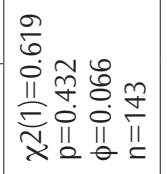 & & 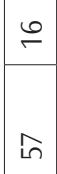 & $\stackrel{\dot{m}}{ }$ & 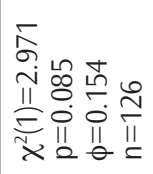 & 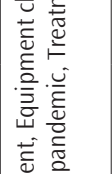 \\
\hline 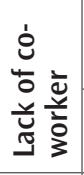 & $\stackrel{\Xi}{\nu}$ & 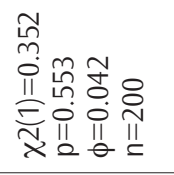 & & $\hat{m}$ & $\stackrel{\infty}{\leftarrow}$ & 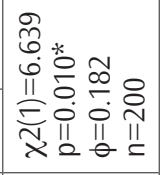 & & $\stackrel{\stackrel{\sim}{\sim}}{\sim}$ & $\approx$ & 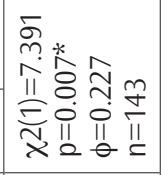 & & $\bar{v}$ & 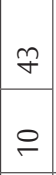 & 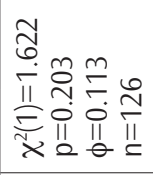 & 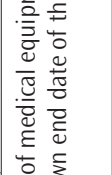 \\
\hline 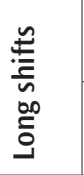 & $\stackrel{\tilde{\nu}}{\nu}$ & 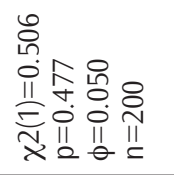 & & \& & $\stackrel{\nu}{ }$ & 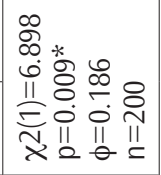 & & $\grave{\sim}$ & $\approx$ & 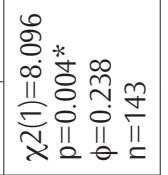 & & $\stackrel{m}{2}$ & $\stackrel{\infty}{\sim}$ & 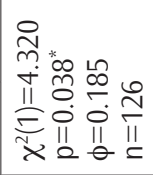 & 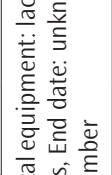 \\
\hline 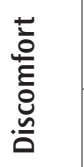 & $\stackrel{\tilde{\nu}}{\tilde{\nu}}$ & 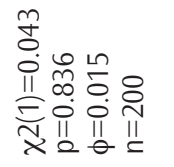 & & $N$ & 8 & 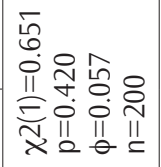 & & 8 & $\mp$ & 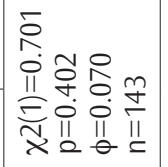 & & 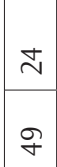 & $m$ & 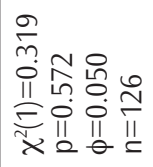 & 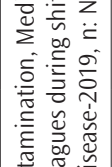 \\
\hline$\frac{u}{a}$ & $\stackrel{\check{\nu}}{\nu}$ & 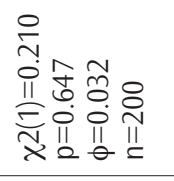 & & $\stackrel{\llcorner}{\forall}$ & 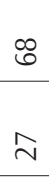 & 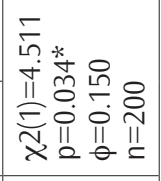 & & $\bar{m}$ & 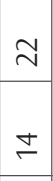 & 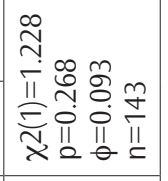 & & $\stackrel{i}{ }$ & $\stackrel{\infty}{\sim}$ & 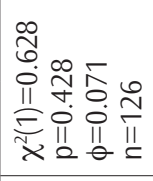 & 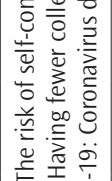 \\
\hline 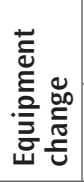 & $\stackrel{\check{\nu}}{\tilde{\nu}}$ & 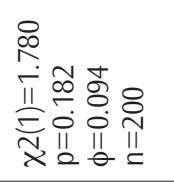 & & $\bar{\sigma}$ & $\stackrel{\infty}{+}$ & 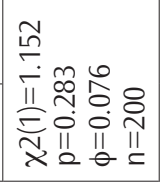 & & $\tilde{n}$ & $\approx$ & 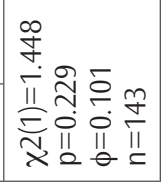 & & $\stackrel{m}{m}$ & $\hat{\nu}$ & 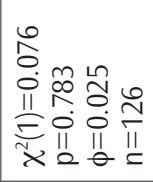 & 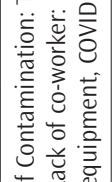 \\
\hline 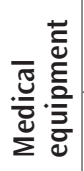 & $\stackrel{\Xi}{\nu}$ & 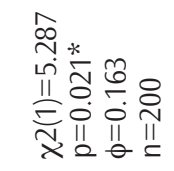 & & $\stackrel{m}{m}$ & $\approx$ & 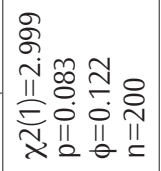 & & $\infty$ & $\underset{\sim}{\Xi}$ & 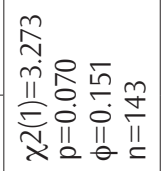 & & in & 눔 & 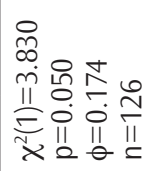 & 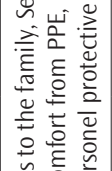 \\
\hline 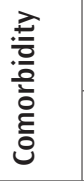 & $\stackrel{\tilde{\nu}}{\nu}$ & 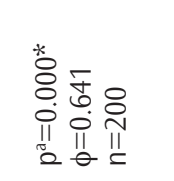 & & 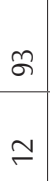 & $\stackrel{\circ}{\circ}$ & 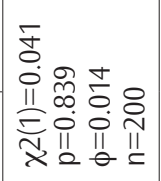 & & 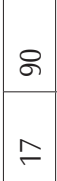 & $m$ & 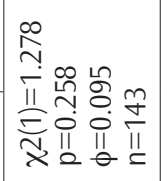 & & ๑ & 迎 & 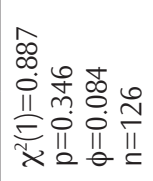 & 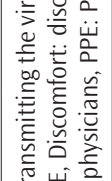 \\
\hline 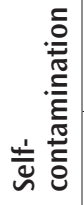 & $\stackrel{\tilde{\nu}}{\nu}$ & 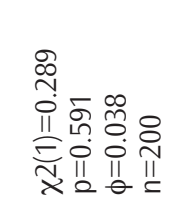 & & $\stackrel{\infty}{\wedge}$ & L & 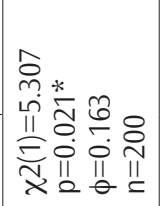 & & $\stackrel{m}{m}$ & $\stackrel{m}{\sim}$ & 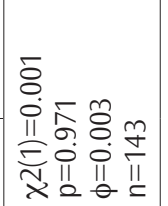 & $\begin{array}{l}\overline{\mathfrak{J}} \\
\stackrel{0}{\tilde{n}} \\
\tilde{y}\end{array}$ & $\tilde{m}$ & $\stackrel{\llcorner}{\sim}$ & 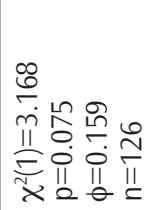 & 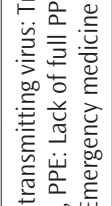 \\
\hline 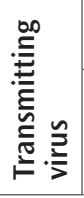 & $\stackrel{\tilde{u}}{\nu}$ & 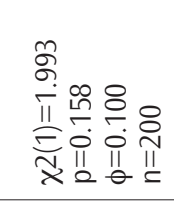 & 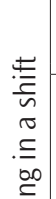 & $\curvearrowright$ & $\stackrel{m}{\wedge}$ & 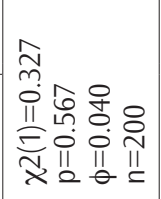 & & 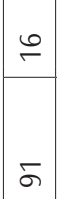 & $\circ$ & 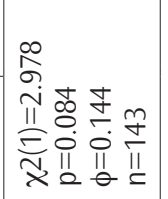 & 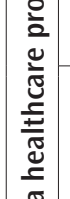 & 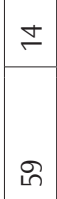 & $\forall$ & 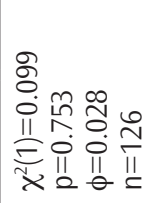 & 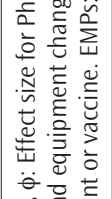 \\
\hline$\frac{\frac{\pi}{0}}{\frac{0}{\frac{\pi}{5}}}$ & & 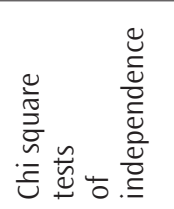 & 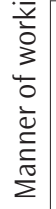 & $\frac{\bar{o}}{\bar{\alpha}}$ & $\begin{array}{l}\sum_{m}^{n} \\
\frac{n}{\infty} \\
\frac{c}{3}\end{array}$ & 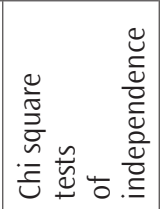 & 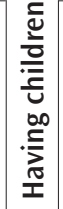 & $\stackrel{\Perp}{\nu}$ & $\stackrel{2}{z}$ & 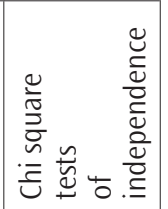 & 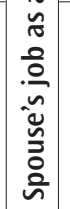 & $\stackrel{\varrho}{\nu}$ & z & & 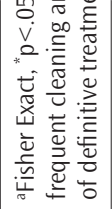 \\
\hline
\end{tabular}


Table 4. Relationship between demographic variables and factors stated by EMPs as effective on coping with stress during CoVID-19 pandemic

\begin{tabular}{|c|c|c|c|c|c|c|c|c|c|c|c|c|c|c|}
\hline \multirow{2}{*}{ Variables } & \multicolumn{2}{|c|}{ Religion } & \multicolumn{2}{|c|}{$\begin{array}{l}\text { Additional } \\
\text { income }\end{array}$} & \multicolumn{2}{|l|}{$\begin{array}{l}\text { Full } \\
\text { PPE }\end{array}$} & \multicolumn{2}{|c|}{ Appreciation } & \multicolumn{2}{|c|}{$\begin{array}{l}\text { Psychological } \\
\text { support }\end{array}$} & \multicolumn{2}{|c|}{$\begin{array}{l}\text { Adequate } \\
\text { rest }\end{array}$} & \multicolumn{2}{|c|}{$\begin{array}{l}\text { Leisure } \\
\text { activities }\end{array}$} \\
\hline & Yes & No & Yes & No & Yes & No & Yes & No & Yes & No & Yes & No & Yes & No \\
\hline Female & 12 & 76 & 44 & 44 & 40 & 48 & 35 & 53 & 8 & 80 & 29 & 59 & 51 & 37 \\
\hline Male & 10 & 102 & 52 & 60 & 66 & 46 & 48 & 64 & 10 & 102 & 45 & 67 & 73 & 39 \\
\hline \multicolumn{15}{|l|}{ Marital status } \\
\hline Married & 12 & 114 & 63 & 63 & 69 & 57 & 50 & 76 & 11 & 115 & 47 & 79 & 79 & 47 \\
\hline Single & 10 & 64 & 33 & 41 & 37 & 37 & 33 & 41 & 7 & 67 & 27 & 47 & 45 & 29 \\
\hline \multicolumn{15}{|c|}{ After-shift accommodation } \\
\hline Alone & 9 & 68 & 37 & 40 & 43 & 34 & 36 & 41 & 10 & 67 & 32 & 45 & 48 & 29 \\
\hline With family & 13 & 110 & 59 & 64 & 63 & 60 & 47 & 76 & 8 & 115 & 42 & 81 & 76 & 47 \\
\hline $\begin{array}{l}\text { Chi-square tests } \\
\text { of independence }\end{array}$ & \multicolumn{2}{|c|}{$\begin{array}{l}\chi 2(1)=0.061 \\
p=0.806 \\
\phi=0.017 \\
n=200\end{array}$} & \multicolumn{2}{|c|}{$\begin{array}{l}\chi 2(1)=0.000 \\
p=0.991 \\
\phi=0.001 \\
n=200\end{array}$} & $\begin{array}{l}\chi 2(1 \\
p=0 \\
\phi=0 . \\
n=2\end{array}$ & 407 & $\begin{array}{l}\chi 2(1 \\
p=0 \\
\phi=0 \\
n=2\end{array}$ & $\begin{array}{l}1.423 \\
3 \\
4\end{array}$ & $\begin{array}{l}\chi 2(1) \\
p=0 . \\
\phi=0 . \\
n=20\end{array}$ & $\begin{array}{l}=2.430 \\
119 \\
110 \\
0\end{array}$ & $\begin{array}{l}\chi 2(1 \\
p=0 . \\
\phi=0 . \\
n=2\end{array}$ & $\begin{array}{l}1^{.116} \\
5\end{array}$ & $\begin{array}{l}\chi 2(1 \\
p=0 . \\
\phi=0 . \\
n=2\end{array}$ & $\begin{array}{l}=0.006 \\
938 \\
006 \\
0\end{array}$ \\
\hline Smoking behavior & & & & & & & & & & & & & & \\
\hline Yes & 6 & 63 & 38 & 31 & 38 & 31 & 31 & 38 & 8 & 61 & 30 & 39 & 44 & 25 \\
\hline Yes & 6 & 22 & 17 & 11 & 14 & 14 & 15 & 13 & 6 & 22 & 7 & 21 & 17 & 11 \\
\hline No & 16 & 156 & 79 & 93 & 92 & 80 & 68 & 104 & 12 & 160 & 67 & 105 & 107 & 65 \\
\hline $\begin{array}{l}\text { Chi-square tests } \\
\text { of independence }\end{array}$ & $\begin{array}{l}\mathrm{p}^{\mathrm{a}}=0 \\
\phi=0 \\
\mathrm{n}=2\end{array}$ & & $\begin{array}{l}\chi 2(1 \\
p=0 . \\
\phi=0 . \\
n=2\end{array}$ & $\begin{array}{l}2.109 \\
46 \\
03\end{array}$ & $\begin{array}{l}\chi 2(1 \\
p=0 \\
\phi=0 \\
n=2\end{array}$ & & $\begin{array}{l}\chi 2(1 \\
p=0 \\
\phi=0 \\
n=2\end{array}$ & $\begin{array}{l}1.954 \\
2 \\
9\end{array}$ & $\begin{array}{l}\mathrm{p}^{\mathrm{a}}=0 \\
\phi=0 . \\
\mathrm{n}=2(\end{array}$ & $\begin{array}{l}.025^{*} \\
175 \\
0\end{array}$ & $\begin{array}{l}\chi 2(1 \\
p=0 . \\
\phi=0 . \\
n=2\end{array}$ & $\begin{array}{l}.011 \\
6 \\
0\end{array}$ & $\begin{array}{l}\chi 2(1 \\
p=0 . \\
\phi=0 . \\
n=2\end{array}$ & $\begin{array}{l}=0.023 \\
880 \\
011 \\
0\end{array}$ \\
\hline Manner of workin & & & & & & & & & & & & & & \\
\hline Alone & 9 & 96 & 46 & 59 & 57 & 48 & 39 & 66 & 7 & 98 & 39 & 66 & 64 & 41 \\
\hline with $\mathrm{EMP}(\mathrm{s})$ & 13 & 82 & 50 & 45 & 49 & 46 & 44 & 51 & 11 & 84 & 35 & 60 & 60 & 35 \\
\hline $\begin{array}{l}\text { Chi-square tests } \\
\text { of independence }\end{array}$ & $\begin{array}{l}\chi 2(1 \\
p=0 \\
\phi=0 \\
n=2\end{array}$ & & $\begin{array}{l}\chi 2(1 \\
\mathrm{p}=0 . \\
\phi=0 . \\
\mathrm{n}=2\end{array}$ & $\begin{array}{l}1.555 \\
12 \\
88\end{array}$ & $\begin{array}{l}\chi 2(1 \\
\mathrm{p}=0 . \\
\phi=0 \\
\mathrm{n}=2\end{array}$ & & $\begin{array}{l}\chi 2(1 \\
p=0 \\
\phi=0 \\
n=2\end{array}$ & $\begin{array}{l}1.729 \\
99 \\
3\end{array}$ & $\begin{array}{l}\chi 2(1) \\
\mathrm{p}=0 . \\
\phi=0 . \\
\mathrm{n}=2(\end{array}$ & $\begin{array}{l}=1.469 \\
225 \\
086 \\
0\end{array}$ & $\begin{array}{l}\chi 2(1 \\
\mathrm{p}=0 . \\
\phi=0 . \\
\mathrm{n}=2\end{array}$ & $\begin{array}{l}.002 \\
5 \\
3\end{array}$ & $\begin{array}{l}\chi 2(1 \\
p=0 \\
\phi=0 . \\
n=2\end{array}$ & $\begin{array}{l}=0.103 \\
748 \\
023 \\
0\end{array}$ \\
\hline Having children & & & & & & & & & & & & & & \\
\hline Yes & 14 & 93 & 52 & 55 & 58 & 49 & 49 & 58 & 9 & 98 & 42 & 65 & 70 & 37 \\
\hline No & 1 & 35 & 17 & 19 & 17 & 19 & 8 & 28 & 2 & 34 & 12 & 24 & 21 & 15 \\
\hline
\end{tabular}


Table 4. continued

\begin{tabular}{|c|c|c|c|c|c|c|c|c|c|c|c|c|c|c|}
\hline \multirow{2}{*}{ Variables } & \multicolumn{2}{|c|}{ Religion } & \multicolumn{2}{|c|}{$\begin{array}{l}\text { Additional } \\
\text { income }\end{array}$} & \multicolumn{2}{|c|}{$\begin{array}{l}\text { Full } \\
\text { PPE }\end{array}$} & \multicolumn{2}{|c|}{ Appreciation } & \multicolumn{2}{|c|}{$\begin{array}{l}\text { Psychological } \\
\text { support }\end{array}$} & \multicolumn{2}{|c|}{$\begin{array}{l}\text { Adequate } \\
\text { rest }\end{array}$} & \multicolumn{2}{|c|}{$\begin{array}{l}\text { Leisure } \\
\text { activities }\end{array}$} \\
\hline & Yes & No & Yes & No & Yes & No & Yes & No & Yes & No & Yes & No & Yes & No \\
\hline Yes & 5 & 68 & 36 & 37 & 38 & 35 & 36 & 37 & 5 & 68 & 29 & 44 & 45 & 28 \\
\hline \multirow[t]{2}{*}{ No } & 7 & 46 & 27 & 26 & 31 & 22 & 14 & 39 & 6 & 47 & 18 & 35 & 34 & 18 \\
\hline & \multicolumn{2}{|c|}{$\begin{array}{l}\chi 2(1)=1.441 \\
p=0.230 \\
\phi=0.107 \\
n=126\end{array}$} & \multicolumn{2}{|c|}{$\begin{array}{l}\chi 2(1)=0.033 \\
p=0.857 \\
\phi=0.016 \\
n=126\end{array}$} & \multicolumn{2}{|c|}{$\begin{array}{l}\chi 2(1)=0.513 \\
p=0.474 \\
\phi=0.064 \\
n=126\end{array}$} & \multicolumn{2}{|c|}{$\begin{array}{l}\chi 2(1)=6.728 \\
p=0.009 * \\
\phi=0.231 \\
n=126\end{array}$} & \multicolumn{2}{|c|}{$\begin{array}{l}p^{a}=0.525 \\
\phi=0.078 \\
n=126\end{array}$} & \multicolumn{2}{|c|}{$\begin{array}{l}\chi 2(1)=0.436 \\
p=0.509 \\
\phi=0.059 \\
n=126\end{array}$} & \multicolumn{2}{|c|}{$\begin{array}{l}\chi 2(1)=0.083 \\
p=0.774 \\
\phi=0.026 \\
n=126\end{array}$} \\
\hline
\end{tabular}

${ }^{a}$ Fisher's Exact, " $p<.05$, $\phi$ : Effect size for Phi, religion: Religious believes, Full PPE: Having full personal protective equipment while working, Appreciation: Getting public appreciation, psychological support: Getting psychological support, adequate rest: Having adequate sleep/rest, Additional income: Having additional income, EMPs: Emergency medicine physicians, PPE: personel protective equipment, COVID-19: Coronavirus disease-2019, n: Number

\section{Discussion}

Research findings revealed that EMPs use mostly problemoriented approach in combating the stress and mainly used social support, self-confident, optimistic, helpless, and submissive approaches, respectively. Other studies indicate that healthcare professionals use a self-confident approach more to deal with stress $(16,17)$. In this research, it was found that EMPs used the most social support seeking approach. This may be due to the general stress caused by working in the field of health, as well as the stress factors brought by the COVID-19 pandemic. Sagar et al. (18) state that individuals can tend to combat stress through social support when there is not much to interfere with the source of stress. Besides, the increasing social support of society may have reinforced this trend. In many countries such as the United States of America and Turkey, expressing their feelings of gratitude to healthcare professionals and providing social support well-attended events, such as certain times of applause on the balconies, were organized through social media $(19,20)$. As a matter of fact, research findings in the literature emphasize the relationship between perceived social support seeking and active coping approach to stress (21-23). It is stated that the approach to seeking social support triggers the feeling of sympathy, increases social resources, and reduces the sense of Ioneliness (24). Also, the influential social support offered during and after stressful situations increases the psychological resilience and work performance (25). In a study conducted during the COVID-19 pandemic, it was concluded that there was a negative relationship between the social support level perceived by healthcare professionals and their stress levels (26). There are also studies that examine healthcare professionals' approach to cope with stress supporting the research findings $(27,28)$.
The factors that EMPs stated in this research to cause stress in the COVID-19 pandemic were similar to the study results investigating stress factors felt by the healthcare professionals during the MERS-CoV epidemic (29). Also, similar outcomes were found in another study examining healthcare professionals' stress factors and managers' expectations in the COVID-19 pandemic (30). Furthermore, in a study conducted with healthcare professionals during the COVID-19 pandemic in China, it was found that they perceived transmitting the virus to their families and lack of PPE as a stress factor (31).

Research findings show a significant difference between men and women in favor of men in terms of their approach to dealing with stress by problem-oriented and optimistic approaches. Sinha and Latha suggest that this difference in women and men's approach to coping with stress may be due to the interaction of sex hormones with adrenaline, noradrenaline, and cortisol, which are the three major stress hormones (32). There are studies in the literature revealing that men are more optimistic than women (33). Besides, it is stated that optimists tend to use more problem-oriented coping strategies than pessimists (34). This research finding is compatible with the results of the active approach $(32,35)$, and the optimistic approach findings among men and women healthcare professionals in favor of the problem (36).

Additionally, stress factors, lack of medical equipment, the necessity of frequent cleaning and equipment change, and the lack of definitive treatment and vaccine, the unknown end date of the pandemic perceived by EMPs were found related to gender. Folkman and Lazarus state that the coping approaches of women and men towards emotion do not differ in similar contexts of life, but they differ when it comes to the context in which stress occurs (12). 


\begin{tabular}{|c|c|c|c|c|c|c|c|c|c|}
\hline \multirow{2}{*}{$\begin{array}{l}\text { Coping } \\
\text { with stress } \\
\text { approaches }\end{array}$} & \multirow{2}{*}{\multicolumn{2}{|c|}{ Model }} & \multicolumn{2}{|c|}{ Unstandardized coefficients } & \multirow{2}{*}{$\begin{array}{l}\text { Standardized } \\
\text { coefficients } \\
\text { Beta }\end{array}$} & \multirow{2}{*}{$\mathrm{t}$} & \multirow{2}{*}{ p-value } & \multicolumn{2}{|l|}{$95.0 \% \mathrm{Cl}$} \\
\hline & & & B & $\begin{array}{l}\text { Std. } \\
\text { Error }\end{array}$ & & & & $\begin{array}{l}\text { Lower } \\
\text { bound }\end{array}$ & $\begin{array}{l}\text { Upper } \\
\text { bound }\end{array}$ \\
\hline \multirow{6}{*}{$\begin{array}{l}\text { Problem- } \\
\text { oriented } \\
\text { approach }\end{array}$} & \multirow[t]{2}{*}{1} & Constant & 64.605 & 1,479 & & 43.693 & 0.000 & 61.690 & 67.521 \\
\hline & & Full PPE (yes) & -5.250 & 2,031 & -0.181 & -2.585 & $0.010^{*}$ & -9.255 & -1.245 \\
\hline & \multirow{4}{*}{2} & Constant & 66.073 & 1,609 & & 41.059 & 0.000 & 62.900 & 69.247 \\
\hline & & Full PPE (yes) & -4.809 & 2,022 & -0.165 & -2.379 & $0.018^{*}$ & -8.796 & -0.822 \\
\hline & & Adequate rest (yes) & -4.599 & 2,090 & -0.153 & -2.201 & $0.029 *$ & -8.720 & -0.478 \\
\hline & & \multicolumn{8}{|c|}{$R=0.236, R^{2}=0.056, F(2,197)=5.827, p=0.003$, Durbin Watson=2.160, VIF=1.010 } \\
\hline \multirow{3}{*}{$\begin{array}{l}\text { Self- } \\
\text { confident } \\
\text { approach }\end{array}$} & \multirow[t]{2}{*}{1} & Constant & 67.173 & 1.758 & & 38.206 & 0.000 & 63.706 & 70.640 \\
\hline & & Full PPE & -8.593 & 2.415 & -0.245 & -3.558 & $0.000^{*}$ & -13.355 & -3.830 \\
\hline & & \multicolumn{8}{|c|}{$R=0.245, R^{2}=0.060, F(1,198)=12.659, p=0.000$, Durbin Watson=2.1, VIF=1 } \\
\hline \multirow{3}{*}{$\begin{array}{l}\text { Optimistic } \\
\text { approach }\end{array}$} & & Constant & 60.847 & 1.720 & & 35.369 & 0.000 & 57.454 & 64.239 \\
\hline & & Adequate rest (yes) & -6.522 & 2.828 & -0.162 & -2.306 & 0.022 & -12.100 & -0.945 \\
\hline & & \multicolumn{8}{|c|}{$R=0.162, R^{2}=0.026, F(1,198)=5.318, p=0.022$. Durbin Watson=2, VIF=1 } \\
\hline \multirow{3}{*}{$\begin{array}{l}\text { Social } \\
\text { support } \\
\text { seeking } \\
\text { approach }\end{array}$} & & Constant & 67.147 & 1.702 & & 39.445 & 0.000 & 63.790 & 70.504 \\
\hline & & Additional income (yes) & -5.168 & 2.457 & -0.148 & -2.103 & $0.037 *$ & -10.014 & -0.323 \\
\hline & & \multicolumn{8}{|c|}{$R=0.148, R^{2}=0.022, F(1,198)=4.424, p=0.037$, Durbin Watson=1.9, VIF=1 } \\
\hline \multirow{10}{*}{$\begin{array}{l}\text { Emotion- } \\
\text { oriented } \\
\text { approach }\end{array}$} & \multirow[t]{2}{*}{1} & Constant & 28.526 & 2.213 & & 12.888 & 0.000 & 24.161 & 32.890 \\
\hline & & End date (yes) & 6.191 & 2.573 & 0.169 & 2.406 & $0.017^{*}$ & 1.117 & 11.265 \\
\hline & \multirow{3}{*}{2} & Constant & 25.934 & 2.481 & & 10.454 & 0.000 & 21.042 & 30.826 \\
\hline & & End date (yes) & 6.456 & 2.550 & 0.176 & 2.531 & $0.012^{*}$ & 1.427 & 11.485 \\
\hline & & Additional income (yes) & 4.991 & 2.239 & 0.155 & 2.229 & $0.027 *$ & 0.576 & 9.407 \\
\hline & 3 & Constant & 23.755 & 2.652 & & 8.958 & 0.000 & 18.525 & 28.984 \\
\hline & & End date (yes) & 5.999 & 2.535 & 0.163 & 2.367 & $0.019^{*}$ & 1.000 & 10.998 \\
\hline & & Additional income (yes) & 4.864 & 2.219 & 0.151 & 2.192 & $0.030 *$ & 0.488 & 9.239 \\
\hline & & Full PPE (yes) & 4.866 & 2.226 & 0.151 & 2.186 & $0.030^{*}$ & 0.476 & 9.255 \\
\hline & & $\mathrm{R}=0.274, \mathrm{R}^{2}=0.075, \mathrm{~F}(3,1$ & $6)=5.288$ & Durbin Wa & $\mathrm{O} O \mathrm{n}=1.9, \mathrm{VIF}=1-$ & 009 & & & \\
\hline \multirow{10}{*}{$\begin{array}{l}\text { Helpless } \\
\text { approach }\end{array}$} & \multirow[t]{2}{*}{1} & Constant & 27.644 & 2.487 & & 11.115 & 0.000 & 22.739 & 32.549 \\
\hline & & End date (yes) & 7.970 & 2.891 & 0.192 & 2.756 & $0.006^{*}$ & 2.268 & 13.671 \\
\hline & \multirow{3}{*}{2} & Constant & 24.600 & 2.784 & & 8.835 & 0.000 & 19.108 & 30.091 \\
\hline & & End date (yes) & 8.280 & 2.863 & 0.200 & 2.893 & $0.004^{*}$ & 2.635 & 13.925 \\
\hline & & Additional income (yes) & 5.864 & 2.513 & 0.161 & 2.333 & $0.021^{*}$ & 0.908 & 10.820 \\
\hline & \multirow{5}{*}{3} & Constant & 22.069 & 2.974 & & 7.421 & 0.000 & 16.204 & 27.934 \\
\hline & & End date (yes) & 7.750 & 2.843 & 0.187 & 2.726 & $0.007^{*}$ & 2.144 & 13.356 \\
\hline & & Additional income (yes) & 5.715 & 2.488 & 0.157 & 2.297 & $0.023 *$ & 0.808 & 10.623 \\
\hline & & Full PPE (yes) & 5.649 & 2.496 & 0.155 & 2.263 & $0.025^{*}$ & 0.726 & 10.572 \\
\hline & & \multicolumn{8}{|c|}{$R=0.294, R^{2}=0.087, F(3,196)=6.203, p=0.000$, Durbin Watson=1.8, VIF=1-1.009 } \\
\hline
\end{tabular}


Research results show a significant difference between married and single EMPs in favor of married people in terms of passive and helpless coping approaches to stress. The risk of transmitting the virus to their families, long shifts, lack of full PPE, and lack of medical equipment was related to marital status. There is evidence that satisfaction with the workplace's physical conditions decreases, the helpless approach to coping with stress increases in individuals (14). It is stated that individuals tend towards passive and helpless coping strategies when they feel that the situation is unchangeable and that control is not in their hands $(14,37)$. Also, the loss of beliefs that they can manage the process in this stressful situation, seeing themselves as the cause of the negativities, may cause them to fail to produce a solution to the problem and take a helpless approach (14). It is suggested assuring care of healthcare professionals' family members would enhance workforce confidence and availability (38). However, no significant difference was found between married and single EMPs in terms of problem-oriented stress coping approaches, partially overlaps with other research findings in the literature (17).

It was seen that the helpless stress approaches of smoker EMPs were higher than non-smokers. Besides, the necessity of frequent cleaning and equipment change as a stress factor was related to smoking behavior. This may be because smoking has a shortterm and temporary function that relieves stress. Mansouri et al. (39) found significant positive relationships between the number of cigarettes smoked per day and escape/avoidance, distancing behaviors, which are passive stress approaches to emotions. Additionally, the comorbidity factor and lack of medical equipment were related to having a chronic disease. At this point, EMPs may be trying to suppress the feeling of helplessness they experience in the face of stress factors brought about by the COVID-19 pandemic, which is not yet fully controlled. Also, the fact that smoking is a preventive factor in the treatment of COVID-19 may lead those who are currently smoking to feel themselves at higher risk and lead to an inevitable acceptance in the face of current stress. Indeed, there is evidence in the literature that reveals the link between smoking and negative outcomes of the COVID-19 treatment (40).

Research results suggest that the submissive stress approach of EMPs that remain with their family after their shift is significantly higher than those who stay alone. Besides, after-shift accommodation was related with the risk of transmitting the virus to their families, long shifts and having fewer colleagues in shifts. In the COVID-19 pandemic, public guesthouses and hotels are put into service for the after-shift stays of healthcare professionals to reduce the possibility of transmitting the virus to their families (41). However, despite this opportunity, those who have children or parents looking after may have to stay in their homes after their shifts. Besides, having children was found related to long shifts and having fewer colleagues in shifts. Also, having a spouse work as a healthcare professional was related long shifts. This can be explained by the fact that EMPs cannot find time and energy to share with their children due to increased workload and decreased rest periods. Prolonged shifts can prevent the individual from fulfilling his responsibilities regarding child care, household chores, and shopping (42). In this case, the individual may adopt a fatalistic attitude and accept to experience stressrelated negativities and take a submissive approach (14).

It was found that working alone in a shift was associated with perceiving the risk of self-contamination, long shifts, lack of full PPE, and lack of co-workers. This may be related to the more fatigue of working alone, increased virus load and relaxation in the measures taken, or the lack of time to take the necessary precautions and the necessary professional support. It is stated that working alone increases mental and physical workload and psychosocial risks (43). At this point, it can be noted that dealing with irrefutable personal needs of healthcare professionals such as adequate rest and care of elderly family members in the COVID-19 pandemic will help maintain their individual and team performance in this marathon (38).

Results of this research partially coincide with the findings of the study conducted during the MERS-CoV epidemic period regarding the factors that were stated to be effective in coping with stress in healthcare professionals (29). It is observed that one of the sources of healthcare professionals' work-related stress before COVID-19 pandemic is not being appreciated. In the COVID-19 pandemic period, appreciation of the EMPs was found to be among the factors they stated to cope with stress effectively. This highlights a critical point in showing the change in society's approach to healthcare professionals. The appreciation was found significantly related to the situation of having children and spouse being a healthcare professional. This may be related to the appreciation of healthcare professionals' devoted efforts in the pandemic by society, being a role model for their children, and the satisfying aspect of winning the community's praise in their children's eyes. Likewise, since the spouse is also a healthcare professional, sharing the same difficulty, struggle and appreciation process can be effective in the EMP's coping with stress as a social support factor. In another study, it was found that having a spouse working in the same area and knowing the content of the spouse's work, is beneficial to both to share information and to understand the negativities of the job and to find solutions (44).

Getting psychological support was found related with having a chronic disease. This finding may be related to those with 
chronic disease taking a more pessimistic, fatalistic and passive approach to cope with the COVID-19 pandemic. Studies show that when healthcare professionals experience physiological or psychological health problems, they prefer self-treatment rather than consulting a physician (45). Those who do not have a chronic disease may be more willing and diligent to get psychological support from their social circles or professionals in coping with stress with a more optimistic approach.

The research findings showed that the variables of having full PPE while working and having sufficient sleep and rest were significant predictors of active approach attitudes towards the problem used by EMPs to deal with stress. When analyzed in terms of sub-dimensions, it was determined that having full PPE while working predicted the self-confident approach and having sufficient sleep and rest predicted the optimistic approach. Indeed, other research results reveal that sleep quality is an essential predictor of the stress experienced by healthcare workers in the COVID-19 pandemic (26).

According to this, having full PPE while trying to reduce the risk of virus transmission can reinforce EMPs' desire to fight this stressful situation. It can help them to take stronger steps in the fight against COVID-19 with the sense of trust given by taking precautions. Also, having the opportunity to sleep and rest can positively affect the psychological processes by providing the soul and the body to relax and contributing to the individual's attitude towards stress to be more constructive and optimistic. It was concluded that getting additional income significantly predicted the level of EMPs using the social support seeking approach to deal with stress. In the literature, social support's dimension to support needs for concrete needs such as time, money, and labor is called instrumental support $(46,47)$. At this point, it can be said that getting additional income constitutes the instrumental support dimension of EMPs' social support seeking approaches to cope with stress.

Research findings show that the factors of the unknown end date of the pandemic, getting additional income, and having full PPE while working significantly predict the tendencies of EMPs to choose a passive coping approach to stress and emotions. When analyzed in terms of sub-dimensions, none of the variables discussed in the study can predict the submissive approach statistically. It was determined that the factors of the unknown end date of the pandemic, getting additional income, and having full PPE while working are significant predictors of the helpless approach. In this context, the current uncertainty of how long the COVID-19 pandemic will last and when it will end can create a sense of desperation and a lack of control in EMPs participating in the research. Besides, while getting additional income due to the pandemic makes EMPs feel safe, it may also cause them to perceive that getting extra income is not as meaningful and valuable as before the pandemic. Having full PPE while working can make EMPs feel safe against the virus; on the other hand, they may feel helpless in fighting against the virus and have anxiety about the protection without having full PPE.

\section{Study Limitations}

The research was carried out with 200 EMPs working in EDs during the COVID-19 pandemic. The study can be conducted in a larger sample of other healthcare professionals. Also, research data is limited to data collected through a scale to identify survey and stress coping approaches. At this point, semiconstructed interviews can be held with a smaller group selected from the research participants for a more detailed evaluation. The research was conducted with limited demographic features belong to participants. In subsequent studies, the variables such as age, work experience, duration of shifts, number of children, and number of patients in a shift can be examined to cope with stress.

\section{Conclusion}

It has been determined that EMPs use problem-based active approaches the most and the social support seeking approach significantly among them in the fight against stress brought by the COVID-19 pandemic. It is crucial to provide healthcare professionals with the support they need and analyze stress factors. It is recommended to increase the social support provided to healthcare professionals and to offer them more effective resources in response to the social support seeking approach. Based on our finding that the risk of transmitting the virus to EMPs' families as the most stressful factor during the COVID-19 pandemic, practices aimed at protecting the families of healthcare professionals can be introduced. Within the research scope, it was observed that leisure activities were the most effective in the fight against stress brought about by the COVID-19 pandemic. In this context, the duration of shifts should be arranged so that healthcare professionals can allocate time for themselves, and psychological support should also be provided.

\section{Ethics}

Ethics Committee Approval: This study was approved by Düzce University Non-Interventional Clinical Research Ethics Board with the registration number 2020/84.

Informed Consent: Informed consent was obtained from those who volunteered to participate in the study.

Peer-review: Externally peer-reviewed. 


\section{Authorship Contributions}

Concept: M.C.D, A.K.A., Design:M.C.D, A.K.A., Data Collection and/or Processing: M.C.D, A.K.A., Analysis and/or Interpretation: M.C.D, A.K.A., Literature Search: M.C.D, A.K.A.,

Writing: M.C.D, A.K.A.

Conflict of Interest: No conflict of interest was declared by the authors.

Financial Disclosure: The authors declared that this study received no financial support.

\section{References}

1. Cai H, Tu B, Ma J, Chen L, Fu L, Jiang Y, et al. Psychological Impact and Coping Strategies of Frontline Medical Staff in Hunan Between January and March 2020 During the Outbreak of Coronavirus Disease 2019 (COVID19) in Hubei, China. Med Sci Monit. 2020;26:e924171.

2. Shanafelt TD, Mungo M, Schmitgen J, Storz KA, Reeves D, Hayes SN, et al. Longitudinal Study Evaluating the Association Between Physician Burnout and Changes in Professional Work Effort. Mayo Clin Proc. 2016;91:422-31.

3. Kang L, Li Y, Hu S, Chen M, Yang C, Yang BX, et al. The mental health of medical workers in Wuhan, China dealing with the 2019 novel coronavirus. Lancet Psychiatry. 2020;7:e14.

4. Santarone K, McKenney M, Elkbuli A. Preserving mental health and resilience in frontline healthcare workers during COVID-19. Am J Emerg Med. 2020;38:1530-1.

5. Huang J, Liu F, Teng Z, Chen J, Zhao J, Wang X, et al. Care for the Psychological Status of Frontline Medical Staff Fighting Against Coronavirus Disease 2019 (COVID-19). Clin Infect Dis. 2020;71:3268-9.

6. Lai J, Ma S, Wang Y, Cai Z, Hu J, Wei N, et al. Factors Associated With Mental Health Outcomes Among Health Care Workers Exposed to Coronavirus Disease 2019. JAMA Netw Open. 2020;3:e203976.

7. Revicki DA, May HJ, Whitley TW. Reliability and validity of the Work-Related Strain Inventory among health professionals. Behav Med. 1991;17:111-20.

8. Schwartz JE, Pickering TG, Landsbergis PA. Work-related stress and blood pressure: current theoretical models and considerations from a behavioral medicine perspective. J Occup Health Psychol. 1996;1:287-310.

9. Steptoe A, Kivimäki M. Stress and cardiovascular disease. Nat Rev Cardiol. 2012;9:360-70.

10. Salyers MP, Bonfils KA, Luther L, Firmin RL, White DA, Adams EL, et al. The Relationship Between Professional Burnout and Quality and Safety in Healthcare: A Meta-Analysis. J Gen Intern Med. 2017;32:475-82.

11. Gavin B, Hayden J, Adamis D, McNicholas F. Caring for the Psychological WellBeing of Healthcare Professionals in the Covid-19 Pandemic Crisis. Ir Med J. 2020;113:51.

12. Folkman S, Lazarus RS. An analysis of coping in a middle-aged community sample. J Health Soc Behav. 1980;21:219-39.

13. Kasiulevičius V, Šapoka V, Filipavičiūtè R. Sample size calculation in epidemiological studies. Gerontologija. 2006;7:225-31.

14. Şahin NH, Durak A. Stresle başa çıkma tarzları ölçeği: Üniversite öğrencileri için uyarlanması. Turkish Journal of Psychology. 1995;10:56-73.

15. Sarmento RP, Costa V. Confirmatory Factor Analysis--A Case study. arXiv preprint arXiv. 2019;1905.05598. Available from: https://arxiv.org/ abs/1905.05598 [Accessed 21 May 2020].

16. Baysak E, Eroğlu MZ, Utku C,, Kaya B. Evaluation of post traumatic stress disorder, burnout and coping styles in emergency and intensive care unit employees. J Clin Psy. 2019;22:36-47.
17. Şen H, Toygar M, Türker $T$, İne $N$, Tuğcu H, Keskin H. The Evaluation of Techniques for Overcoming with the Stress in a Physician Group. TAF Prev Med Bull. 2012;11:211-8.

18. Sagar SS, Lavallee D, Spray CM. Coping with the effects of fear of failure: A preliminary investigation of young elite athletes. Journal of Clinical Sports Psychology. 2009;3:73-98.

19. Twitter (2020). \#SağlıkcılarınıAlkısla [Online]. Website https://twitter com/search?q=\%23Sa\%C4\%9FI\%C4\%B1 kc\%C4\%B1lar\%C4\%B1n\%C4\%B1Alk $\%$ C4\%B1sla\&src= typed_query [Accessed 21 May 2020].

20. Twitter (2020). \#SolidarityAt8 [Online]. Website https://twitter.com/ SolidarityAt8 [Accessed 21 May 2020].

21. DeLongis A, Holtzman S. Coping in context: the role of stress, social support, and personality in coping. J Pers. 2005;73:1633-56.

22. Dougall AL, Hyman KB, Hayward MC, McFeeley S, Baum A. Optimism and Traumatic Stress: The Importance of Social Support and Coping 1. J Appl Soc Psychol. 2001;31:223-45.

23. Malkoç A, Yalçin I. Relationships among Resilience, Social Support, Coping, and Psychological Well-Being among University Studentss. Turkish Psychological Counseling and Guidance Journal. 2015;5:35-43.

24. Prati G, Pietrantoni L. Optimism, social support, and coping strategies as factors contributing to posttraumatic growth: A meta-analysis. Journal of loss and trauma. 2009;14:364-88.

25. Brooks SK, Rubin GJ, Greenberg N. Traumatic stress within disaster-exposed occupations: overview of the literature and suggestions for the management of traumatic stress in the workplace. Br Med Bull. 2019;129:25-34.

26. Xiao H, Zhang Y, Kong D, Li S, Yang N. The Effects of Social Support on Sleep Quality of Medical Staff Treating Patients with Coronavirus Disease 2019 (COVID-19) in January and February 2020 in China. Med Sci Monit. 2020;26:e923549.

27. Sülemiş I, Dönmez L. Coping Strategies with Stress among Female Staff at a University Hospital. Akd Med J. 2017;1:41-7.

28. Akdemir M, Çetin E, Ersoy M, Polat B, Çulhacı E, Ergün A, et al. The Ways of Coping with the Stress and the Related Factors among Sixth-year Medical Students. Tıp Eğitimi Dünyası. 2015;14:33-43.

29. Khalid I, Khalid TJ, Qabajah MR, Barnard AG, Qushmaq IA. Healthcare Workers Emotions, Perceived Stressors and Coping Strategies During a MERSCoV Outbreak. Clin Med Res. 2016;14:7-14.

30. Shanafelt T, Ripp J, Trockel M. Understanding and Addressing Sources of Anxiety Among Health Care Professionals During the COVID-19 Pandemic. JAMA. 2020;323:2133-4.

31. Chen Q, Liang M, Li Y, Guo J, Fei D, Wang L, et al. Mental health care for medical staff in China during the COVID-19 outbreak. Lancet Psychiatry. 2020;7:e15-e6.

32. Sinha S, Latha GS. Coping response to same stressors varies with gender National J Physiol Pharm Pharmacol. 2018;8:1053-6.

33. Jacobsen B, Lee JB, Marquering W, Zhang CY. Gender differences in optimism and asset allocation. J Econ Behav Org. 2014;107:630-51.

34. Scheier MF, Carver CS, Bridges MW. Optimism, pessimism, and psychological well-being. In E. C. Chang (Ed.) Optimism \& pessimism: Implications for theory, research, and practice . American Psychological Association. 2001. p.189-216.

35. Koinis A, Giannou V, Drantaki V, Angelaina S, Stratou E, Saridi M. The Impact of Healthcare Workers Job Environment on Their Mental-emotional Health. Coping Strategies: The Case of a Local General Hospital. Health Psychol Res. 2015;3:1984.

36. Ergin A, Uzun SU, Bozkurt Ai. The Methods of Coping with the Stress Among Medical Faculty Students and the Relationship between Sociodemographic Characteristics and these Methods. Firat Med J. 2014;19:31-7. 
37. Carver CS, Scheier MF, Weintraub JK. Assessing coping strategies: a theoretically based approach. J Pers Soc Psychol. 1989;56:267-83.

38. Adams JG, Walls RM. Supporting the Health Care Workforce During the COVID-19 Global Epidemic. JAMA. 2020;323:1439-40.

39. Mansouri A, Kavi E, Ahmadpoori SF, Amin E, Bazrafshan MR, Piroozi A, et al. Cigarette Smoking and Coping Strategies with Stress in Young Adults of Larestan. Jundishapur J Health Sci. 2018;11:e83121.

40. Vardavas Cl, Nikitara K. COVID-19 and smoking: A systematic review of the evidence. Tob Induc Dis. 2020;18:20.

41. Republic of Turkey Ministry of Health (2020). New Regulations for Healthcare Workers to Fight Coronavirus [online]. Website https://www.saglik.gov.tr/ EN,64696/new-regulations-for-healthcare-workers-to-fight-coronavirus.html [accessed 21 May 2020].

42. Harrington JM. Health effects of shift work and extended hours of work. Occup Environ Med. 2001;58:68-72.
43. Weerdt CVD, Baratta R. Changes in working conditions for home healthcare workers and impacts on their work activity and on their emotions. Production. 2015;25:344-53.

44. Şener E, Kocaoğlu M, Baybek H, Kıvrak A. Dual Career Couples in the Health Sector: A Qualitative Study with Nurse Couples. Hacettepe Journal of Health Administration. 2018;21:461-72.

45. Töyry S, Räsänen K, Kujala S, Aärimaa M, Juntunen J, Kalimo R, et al. Selfreported health, illness, and self-care among finnish physicians: a national survey. Arch Fam Med. 2000;9:1079-85.

46. Semmer NK, Elfering A, Jacobshagen N, Perrot T, Beehr TA, Norbert B. The emotional meaning of instrumental social support. Int J Stress Manag. 2008;15:235-51.

47. Demaray MK, Malecki CK, Jenkins LN. Social support: How to assess and include it in research on prevention and youth outcomes. In Handbook of youth prevention science: Routledge. 2012. p. 175-88. 